\section{Look to the side (effects)}

\section{By Lauren Martz, Staff Writer}

Researchers at the European Molecular Biology Laboratory have developed an approach for repurposing therapeutics that could eliminate a lot of the guesswork associated with current methods. Their work, published in Science, describes a systematic method to identify alternative drug targets and hence new indications for existing drugs using a comparative analysis of side effects. ${ }^{1}$

Scientists interviewed by SciBX recognized the potential of the approach for identifying new targets, but they pointed out the need to determine whether these 'side-target' effects have therapeutic value or not.

Many repurposing efforts involve screening libraries to find compounds that have similar chemistry or hit the same target as a marketed drug and then validating the activity of any resulting hits in vitro. ${ }^{2}$ The methods are essentially a guessing game, however, and assays are often necessary to screen every compound for the desired chemical and structural properties.

In the Science paper, Peer Bork and colleagues at the European Molecular Biology Laboratory (EMBL) proposed that side effects might actually provide insight into additional targets that drugs act upon, and they predicted that compounds with similar side effects may share targets. Bork is joint unit coordinator and senior scientist in the structural and computational biology unit of EMBL, and he is corresponding author on the paper.

The EMBL team developed a measurement of side-effect similarity between drug pairs by analyzing the listed side effects of 502 marketed drugs with known targets to make correlations. The researchers then analyzed side-effect data on 748 other marketed drugs, including 244 drugs with unknown targets. Using the reference set, they pulled out 2,903 potential drug pairs with common targets.

A key question, said Bork, is "if the novel off targets that are found go together with a beneficial therapeutic effect. We certainly hope that, based on our method, some drugs can be repurposed."

Grant Zimmermann, VP of discovery research at CombinatoRx Inc., noted that although a compound may have previously unknown effects on a given target, "that doesn't necessarily mean that it will have a therapeutic effect. You need to see whether the off-target effects have actual therapeutic benefit."

He also said the selectivity of the compounds for new targets pales in comparison to the selectivity for original targets. For the new targets he said, "the binding for all the inhibitory concentration curves are in the 10-100 micromolar range. This is a problem because to leverage the effects of the off target, you are certainly going to be using a lot of the agent and will be taking on at least the full original therapeutic effect."

David Cavalla, founder and both commercial and general manager of Arachnova Ltd., noted that the paper does not address "the selectivity for the new indication relative to the original indication." Because of the relatively weak binding, he said, the method might best be used to identify leads directed at the off targets that could be further refined by using medicinal chemistry.

Arachnova’s SwitchBase database links approved and clinical-stage drugs with existing indications and new indications based on scientific literature about the compounds.

\section{Driving toward automation}

Target selectivity aside, researchers agreed that side-effect analysis is a relatively simple way to identify candidates that might otherwise be overlooked for repurposing. However, another common theme among researchers contacted by SciBX was the desire for automation of the technique.

"The advantages are clear: using existing information, they offer up a testable hypothesis for identifying drug targets," said Corey Nislow, assistant professor in the Banting and Best Department of Medical Research and the Department of Molecular Genetics at the University of Toronto.

Zimmermann told SciBX the paper validates one of CombinatoRx's key presumptions. "The one-drug, one-target paradigm is not true," he said. "Most [drugs] hit many targets and have many effects we aren't aware of. These include side-effect issues that we don't want, but also new uses."

CombinatoRx is developing combinations of approved drugs using its combination high throughput screening (cHTS) technology to screen pair-wise combinations from a library of approved drugs. The company's cell-based assays correspond to major diseases, and each one screens for the effects of millions of possible dose-specific combinations of existing drugs in each model.

"There are a whole slew of compounds for which we don't understand the pharmacology. For many, there are theories on what those targets are, but not proof or confirmation," said Srinivas Rao, CSO of Cypress Bioscience Inc. The technique published in Science, he said, "is great for better understanding a given drug and its side effects. It could be used to go back and design better molecules."

Cypress' repurposing strategy involves working backward from an indication of interest to identify compounds in clinical development for other indications that might have an applicable mechanism.

Compared with the brute force method, which involves putting every drug through in vitro and cell-based assay screening, Rao said benefits of the side-effect approach include analyzing how the compounds actually act in the human body.

"Looking at the in vitro effects and properties is a problem because living cells function differently than those in a Petri dish," Rao told SciBX. 
Rao did say the multiple manual steps were the largest problem with the side effect-based reprofiling technique. Nislow agreed and thinks automation will help move the method beyond proof of principal.

Zimmerman noted that the method also has limited efficacy for a drug that has not been on the market for a significant amount of time because there will not be a full picture of its side effects.

Bork told SciBX that the approach has been patented.

Ivica Letunic, $\mathrm{CEO}$ of biobyte solutions $\mathrm{GmbH}$, said the company is in the process of exclusively licensing the IP. The company plans to provide services based on the technology, including side effect-based drug target prediction.

\section{REFERENCES}

1. Campillos, M. et al. Science; published online July 11, 2008; doi:10.1126/science. 1158140

Contact: Peer Bork, European Molecular Biology Laboratory, Heidelberg, Germany

e-mail: bork@embl.de

2. Bisson, W. et al. Proc. Natl. Acad. Sci. USA 104, 11927-11932 (2007)

COMPANIES AND RESEARCH INSTITUTIONS MENTIONED

Arachnova Ltd., Cambridge, U.K.

biobyte solutions $\mathrm{GmbH}$, Heidelberg, Germany

CombinatoRx Inc. (NASDAQ:CRXX), Cambridge, Mass.

Cypress Bioscience Inc. (NASDAQ:CYPB), San Diego, Calif. European Molecular Biology Laboratory, Heidelberg, Germany University of Toronto, Toronto, Ontario, Canada 